\title{
Prevalence, phylogroups, antimicrobial susceptibility and ge- netic diversity of Escherichia coli isolates from food products
}

\author{
Babak Pakbin 1,2, Samaneh Allahyari ${ }^{1}$, Zahra Amani ${ }^{~}$, Wolfram Manuel Brück 2,", Razzagh Mahmoudi 1,* and Amir \\ Peymani 1 \\ 1 Medical Microbiology Research Center, Qazvin University of Medical Sciences, Qazvin, Iran.; \\ b.pakbin@ut.ac.ir; z.amani@ut.ac.ir; samaneh.alahyari66@gmail.com; r.mahmodi@yahoo.com; \\ a.peymani@gmail.com \\ 2 Institute for Life Technologies, University of Applied Sciences Western Switzerland Valais-Wallis, 1950 Sion \\ 2, Switzerland; wolfram.bruck@hevs.ch \\ * Correspondence: wolfram.bruck@hevs.ch; r.mahmodi@yahoo.com
}

\begin{abstract}
Background: The emergence of multi-drug resistant E. coli is an important matter of increasing considerable concern to global public health. The aim of this study was to investigate the incidence, antibiotic resistance pattern, phylogroups and genetic variation of E. coli isolates from raw milk, vegetable salad and ground meat samples. Methods: Culture-based techniques, KirbyBauer disk diffusion susceptibility testing, PCR and RAPD assays were used to determine the incidence rate, antimicrobial resistance pattern, phylogenetic groups and genetic diversity of the $E$. coli isolates. Results: E. coli isolates were highly resistant to amoxicillin (79.16\%), trimethoprimsulfamethoxazole $(70.83 \%)$, amoxicillin-clavulanic acid $(62.50 \%)$, tetracycline $(54.16 \%)$, chloramphenicol $(54.16 \%)$, nitrofurantoin $(54.16 \%)$, ampicillin $(45.83 \%)$, streptomycin $(45.83 \%)$, and kanamycin (33.33\%); and completely susceptible to norfloxacin and azithromycin. $70.83 \%$ of the isolates were multi-drug resistant. Most E. coli isolates (46\%) belonged to phylogroup A. RAPD with UBC245 primer categorized the isolates into 11 clusters. A high level of genetic diversity was found among the isolates; however, $33.3 \%$ of the isolates were grouped in a major cluster (R5). Conclusions: Antibiotic resistance patterns are randomly distributed among the genetic clusters. Novel, practical, efficient food safety control and surveillance systems of multi-drug resistant foodborne pathogens are required to control the foodborne pathogen contamination.
\end{abstract}

Keywords: Escherichia coli; Antimicrobial resistance; Food samples; Phylogenetic group; Genetic diversity

\section{Introduction}

Foodborne diseases are defined as disorders caused by agents (bacteria, fungi, viruses, parasites and chemicals) that are usually either toxic or infectious in nature and enter the human body through the ingestion of food or drinks [1]. Foodborne bacteria are the main microbial factors causing foodborne diseases with significant adverse effects on human health and economic well-being. Foodborne pathogens can induce mild to severe both intestinal and extra-intestinal symptoms in humans [2]. WHO estimated that considered foodborne hazards caused more than 600 million foodborne diseases leading to more than 33 million disability-adjusted life years (DALYs) and 420000 deaths annually worldwide [3]. The global burden of foodborne illnesses is comparable to those of the main infectious diseases such as tuberculosis, HIV and malaria; and certain risk factors including unimproved sanitation, air pollution and dietary risk factors [4]. At least $90 \%$ of all foodborne diseases are caused by diarrheal disease agents, indicating that many diarrheal illnesses are pathologically benign. The most prevalent foodborne pathogens contributing to the global burden of diarrheal illnesses have been reported to be Noroviruses, Campylobacter spp., Escherichia coli, Salmonella spp., Shigella 
spp., Giardia spp., and Entamoeba histolytica. E. coli is one of the main challenges and concerns in food safety and public health [3].

Escherichia coli is gram-negative rod-shaped facultative anaerobic bacteria belonging to the Enterobacteriaceae family. E. coli is a commensal bacterium and typical inhabitant of gastrointestinal tract of warm-blooded animals such as mammals, including human, cattle, and pigs, amongst others [5]. E. coli mostly remains confined to the human or animal gastrointestinal lumen. However, these bacteria can also cause severe intestinal and extraintestinal infections in immunocompromised or debilitated hosts [6]. Diarrheal diseases and extraintestinal infections such as hemorrhagic uremic syndrome (HUS), sepsis and meningitis in both humans and animal are caused by specific groups of E. coli [7]. Nevertheless, these bacteria have been generally regarded as an indicator of human fecal contamination due to it is consistent presence in human feces. Contamination with either pathogenic or non-pathogenic E. coli may occur during any farm-to-table processing steps from animal, environmental or human sources and cause foodborne diseases and outbreaks in human and animals [8]. Investigation of serogroups, pathotypes, sequence types and phylogroups in E. coli strains allow characterization of potential pathogenicity and virulence of the isolates from human, animal, food, water and environments [9].

A classification system based on phylogenetic cluster characterization of E. coli has previously been developed by Clermont et al. (2000) for tracking microbial source, determination of clonal relationship and determination potential pathogenicity among $E$. coli isolates [10]. Phylogenetic analysis of E. coli isolates has indicated that E. coli clones are divided into four main distinct groups, including A, B2, B2 and D; and seven main subgroups consisting of A0, A1, A2, B22, B23, D1 and D2. A refined phylogroup classification has recently been proposed by Clermont et al. (2013), and four more phylogroups including C, E, F and Escherichia cryptic clade I were added [11]. E. coli strains belonging to the phylogroup B2, and a lesser extent to the phylogroup D, are main causes of extraintestinal infections in human. Also, the strains belonging to the phylogroup A are mostly commensal [12]. E. coli strains causing diarrheal diseases are most probably of the phylogroups B2 and E. There is a strong link between the virulence and phylogeny in $E$. coli infections. Phenotypic and genotypic properties of E. coli strains belonging to all phylogroups are entirely different. These properties include sugar fermentation, growth temperature, presence or absence of virulence encoding genes and antibiotic resistance pattern [13].

Antibiotics have a major role in treatment of bacterial infections in humans and animals by decreasing mortality and morbidity associated with the infectious diseases. Consequently, antimicrobial resistance in foodborne pathogens, particularly those identified in animal origin foods have been increased around the world [14]. Several causative factors have been attributed to this increase including use of antibiotics for growth promotion of farm animals, addition of clinical antibiotics to the farm animal feeds, and overuse of antimicrobial agents in humans and animals [15]. Emergence of multidrug resistant (MDR) foodborne pathogens has been considered one of the main concerns in public health [16]. E. coli strains are more likely to be resistant to several classes of antibiotics as a MDR foodborne pathogen. MDR E. coli has been recognized as one of the most significant challenges in food safety [17]. Little is known about phylogroups, antimicrobial susceptibility and genetic relatedness of E. coli strains isolated from food products [18]. The purpose of this study was to determine the prevalence rate, phylogroups, antimicrobial susceptibility patterns and genetic diversity in E. coli strains isolated from food products including vegetable salad, raw cow milk and ground meat samples.

\section{Results}

2.1. Isolation and identification of E. coli in food products

A total of $24(6.95 \%)$ E. coli strains were isolated and confirmed from 345 food samples. Prevalence rates of $E$. coli isolated from raw milk, vegetable salad, and ground meat samples are illustrated in Figure 1. Among all E. coli isolates, $15(54.16 \%)$ of 24, 5 
(20.83\%) of 24 and $4(16.66 \%)$ of 24 isolates were detected in raw cow milk, ground meat and vegetable salad samples, respectively. All E. coli isolates were primarily isolated and detected using culture-based methods and then confirmed by biochemical tests. Prevalence rate of the E. coli isolates was significantly $(P<0.01)$ higher in raw com milk than that in vegetable salad and ground meat samples.

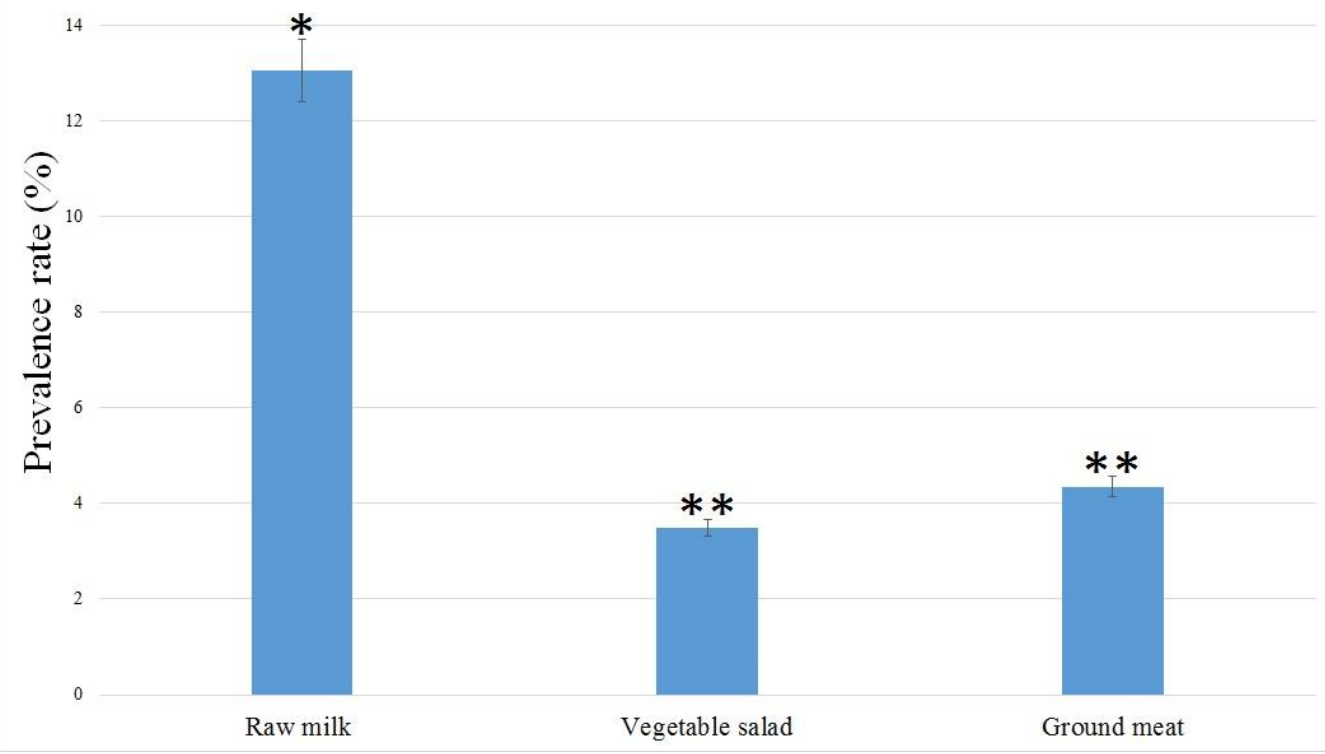

Figure 1. Prevalence rates of E. coli in different food samples. ${ }^{*}$ and ${ }^{* *}$ indicates significant differences $(\mathrm{P}>0.05)$.

\subsection{Antimicrobial susceptibility evaluation the of E. coli isolates}

All twenty-four $E$. coli isolates were tested for their antimicrobial resistance against nine different common classes of antibiotics and nineteen different commercial antibiotics. The results of phenotypic resistance tests to antibiotics of the isolates are shown in Table 1. Amoxicillin (19 isolates; 79.16\%), trimethoprim-sulfamethoxazole (17 isolates; $70.83 \%)$, amoxicillin-clavulanic acid (15 isolates; 62.50\%), tetracycline (13 isolates; $54.16 \%$ ), chloramphenicol (13 isolates; $54.16 \%$ ); nitrofurantoin (13 isolates; $54.16 \%$ ), ampicillin (11 isolates; $45.83 \%$ ); streptomycin (11 isolates; $45.83 \%$ ) and kanamycin (8 isolates; $33.33 \%$ ) resistance were the dominant resistance phenotypes among the E. coli isolates. However, the lowest antibiotic resistance phenotypes were observed against cefoxitin (4 isolates; $16.66 \%$ ), colistin (4 isolates; $16.66 \%$ ), cefepime ( 3 isolates; $12.50 \%$ ), imipenem ( 1 isolate; $4.16 \%$ ), amikacin ( 1 isolate; $4.16 \%$ ), gentamicin (1 isolate; $4.16 \%$ ), nalidixic acid (1 isolate; $4.16 \%$ ) and levofloxacin (1 isolate; $4.16 \%$ ). All E. coli isolates were completely sensitive to norfloxacin and azithromycin. No significant differences were seen among the antibiotic resistant patterns of the E. coli strains isolated from raw milk, vegetable salad, and ground meat samples. 17 of 24 E. coli strains $(70.83 \%)$ expressed resistance to at least three different classes of antimicrobial agents and were considered as MDR E. coli isolates (Table 2). In this study, resistance to five $(20.8 \%)$ or six $(20.8 \%)$ antibiotic classes simultaneously were the most frequent patterns of multidrug resistance for the E. coli isolates. The multidrug resistance pattern of $\beta$-lactams, nitroheterocyclics, aminoglycosides, folate pathway antagonists, lipopeptides, tetracyclines and phenicols was the most resistance profile $(\mathrm{n}=2 ; 8.3 \%)$. In summary, most of the E. coli isolates $(\mathrm{n}=14$; $58.3 \%$ ) were resistant to four, five or six classes of antibiotics simultaneously, whereas, only $4.1 \%(\mathrm{n}=1)$ and $8.3 \%(\mathrm{n}=2)$ of the isolates were simultaneously resistant to three and seven classes of antimicrobial agents, respectively. 
Table 1. Antibiotic resistance phenotype of E. coli isolated from food samples.

\begin{tabular}{|c|c|c|c|c|c|}
\hline \multirow[t]{2}{*}{ Antibiotic class } & \multirow[t]{2}{*}{ Antibiotic Agent } & \multicolumn{4}{|l|}{$\mathrm{n}(\%)$} \\
\hline & & Raw milk $(\mathrm{n}=15)$ & Ground meat $(n=5)$ & Vegetable salad $(\mathrm{n}=4)$ & Total $(\mathrm{n}=24)$ \\
\hline \multirow[t]{6}{*}{$\beta$-Lactams } & cefoxitin & $3(20.0)$ & $0(0)$ & $1(25.0)$ & $4(16.66)$ \\
\hline & imipenem & $1(6.66)$ & $0(0)$ & $0(0)$ & $1(4.16)$ \\
\hline & amoxicillin & $12(80.0)$ & $5(100)$ & $2(50.0)$ & $19(79.16)$ \\
\hline & ampicillin & $8(53.33)$ & $2(40.0)$ & $1(25.0)$ & $11(45.83)$ \\
\hline & cefepime & $2(13.33)$ & $0(0)$ & $1(25.0)$ & $3(12.50)$ \\
\hline & amoxicillin-clavulanic acid & $11(73.33)$ & $3(60.0)$ & $1(25.0)$ & $15(62.50)$ \\
\hline \multirow[t]{4}{*}{ Aminoglycosides } & streptomycin & $7(46.66)$ & $4(80.0)$ & $0(0)$ & $11(45.83)$ \\
\hline & kanamycin & $8(53.33)$ & $0(0)$ & $0(0)$ & $8(33.33)$ \\
\hline & amikacin & $1(6.66)$ & $0(0)$ & $0(0)$ & $1(4.16)$ \\
\hline & gentamicin & $1(6.66)$ & $0(0)$ & $0(0)$ & $1(4.16)$ \\
\hline \multirow[t]{3}{*}{ Quinolones and fluoroquinolones } & nalidixic acid & $0(0)$ & $1(20.0)$ & $0(0)$ & $1(4.16)$ \\
\hline & norfloxacin & $0(0)$ & $0(0)$ & $0(0)$ & $0(0)$ \\
\hline & levofloxacin & $1(6.66)$ & $0(0)$ & $0(0)$ & $1(4.16)$ \\
\hline Macrolides & azithromycin & $0(0)$ & $0(0)$ & $0(0)$ & $0(0)$ \\
\hline Tetracyclines & tetracycline & $10(66.66)$ & $3(60.0)$ & $0(0)$ & $13(54.16)$ \\
\hline Lipopeptides & colistin & $4(26.66)$ & $0(0)$ & $0(0)$ & $4(16.66)$ \\
\hline Phenicols & chloramphenicol & $9(60.0)$ & $3(60.0)$ & $1(25.0)$ & $13(54.16)$ \\
\hline Nitroheterocyclics & nitrofurantoin & $10(66.66)$ & $0(0)$ & $3(75.0)$ & $13(54.16)$ \\
\hline Folate pathway antagonists & trimethoprim-sulfamethoxazole & $12(80.0)$ & $4(80.0)$ & $1(25.0)$ & $17(70.83)$ \\
\hline
\end{tabular}

Table 2. Multidrug resistance class patterns of $E$. coli isolates from food samples.

\begin{tabular}{|c|c|c|}
\hline No. classes of antibiotics & Multidrug resistance patterns ${ }^{a}$ (No. isolates in each pattern) & No. total isolates $(\%)(n=24)$ \\
\hline One & $\beta \operatorname{Ls}(n=3)$ & $3(12.5)$ \\
\hline Two & $\beta$ Ls-NHCs $(n=4)$ & $4(16.6)$ \\
\hline Three & $\beta$ Ls-AGs-FPAs $(n=1)$ & $1(4.1)$ \\
\hline \multirow[t]{4}{*}{ Four } & $\beta$ Ls-TCs-PNs-FPAs $(\mathrm{n}=1)$ & $4(16.6)$ \\
\hline & $\beta$ Ls-LPs-NHCs-FPAs $(\mathrm{n}=1)$ & \\
\hline & $\beta$ Ls-AGs-NHCs-FPAs $(\mathrm{n}=1)$ & \\
\hline & $\beta$ Ls-PNs-NHCs-FPAs $(\mathrm{n}=1)$ & \\
\hline \multirow[t]{3}{*}{ Five } & $\beta$ Ls-AGs-TCs-PNs-FPAs $(\mathrm{n}=3)$ & $5(20.8)$ \\
\hline & AGs-TCs-LPs-PNs-FPAs (n=1) & \\
\hline & $\beta$ Ls-AGs-TCs-NHCs-FPAs $(\mathrm{n}=1)$ & \\
\hline \multirow[t]{2}{*}{ Six } & $\beta$ Ls-AGs-TCs-NHCs-PNs-FPAs $(n=3)$ & $5(20.8)$ \\
\hline & $\beta$ Ls-AGs-TCs-QNs-PNs-FPAs ( $\mathrm{n}=2)$ & \\
\hline \multirow[t]{2}{*}{ Seven } & $\beta$ Ls-AGs-TCs-LPs-NHCs-PNs-FPAs $(\mathrm{n}=2)$ & $2(8.3)$ \\
\hline & $\begin{array}{l}\text { a } \beta \text { Ls, } \beta \text {-Lactams; NHCs, Nitroheterocyclics; } \\
\text { pathway antagonists, LPs, Lipopeptides; TCs, Tetra } \\
\text { olones and fluoroquinolones. } \\
\text { 2.3. Phylogroups of the E. coli isolates } \\
\text { According to phylogenetic grouping of the } 24 \\
5(21 \%), 5(21 \%) \text { and } 3(12 \%) \text { of the isolates were as } \\
\text { A, E, B1 and D, respectively (Figure } 2) \text {. As shown ir } \\
\text { isolates; } 29.16 \%) \text { and E (4 isolates; } 16.66 \%) \text { were the } \\
\text { the E. coli strains isolated from raw milk samples. I } \\
\text { strains were isolated from vegetable salad sample } \\
\text { differences in distribution of phylogenetic groupi } \\
\text { from each food products. }\end{array}$ & $\begin{array}{l}\text { Aminoglycosides; FPAs, Folate } \\
\text { lines; PNs, Phenicols; QNs, Quin- } \\
\text { oli isolates in this study, } 11(46 \%) \text {, } \\
\text { hed to the phylogenetic groups of } \\
\text { ble 3, phylogenetic groups of A ( } \\
\text { st prevalent phylogroups among } \\
\text { and E phylogenetic group E. coli } \\
\text { lso, there are not any significant } \\
\text { among the E. coli strains isolated }\end{array}$ \\
\hline
\end{tabular}


Table 3. Distribution of phylogroups among the E. coli isolates obtained from different food products.

\begin{tabular}{lllll}
\hline Food product & \multicolumn{4}{l}{$\mathrm{n}(\%)$ of the E. coli isolates $(\mathrm{n}=24)$} \\
\cline { 2 - 5 } & $\mathrm{A}$ & $\mathrm{B} 1$ & $\mathrm{D}$ & $\mathrm{E}$ \\
\cline { 2 - 5 } Raw milk & $7(29.16)$ & $2(8.33)$ & $2(8.33)$ & $4(16.66)$ \\
Vegetable salad & $2(8.33)$ & $2(8.33)$ & $0(0)$ & $0(0)$ \\
Ground meat & $2(8.33)$ & $1(4.16)$ & $1(4.16)$ & $1(4.16)$ \\
\hline
\end{tabular}

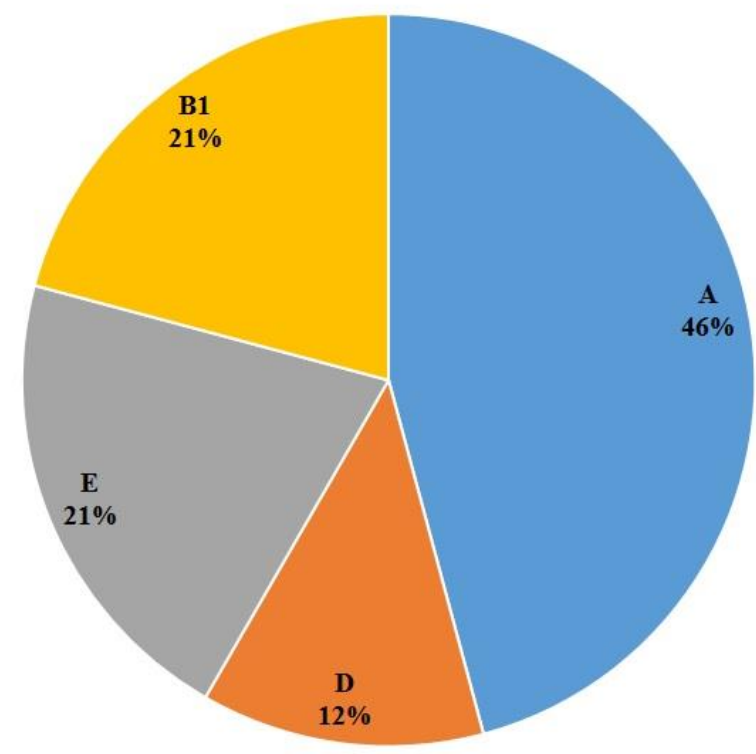

Figure 2. Phylogenetic group distribution of E. coli strains isolated from food samples.

\subsection{Genetic diversity among the E. coli isolates}

At the present study, genetic diversity, and genotypic polymorphism of twentyfour E. coli isolates from raw milk, ground meat and vegetable salad samples were evaluated by RAPD-PCR assay using the UBC245 primer. Amplification of the E. coli isolates with the UBC245 arbitrary oligonucleotide resulted in RAPD-PCR patterns composed of two to seven bands ranged in size from 400 to $>2000$ bp (Figure 3). As shown in Figure 4, UBC245 primer discriminated the twenty-four E. coli isolates into eleven distinct clusters (R1-R11) of genetically identical E. coli isolates with at least 59\% RAPD-PCR profile similarity (a cut-off value at 59\% similarity). All RAPD-PCR groups were compared using $t$ test and the significant differences $(P>0.05)$ were not found. Genetic diversity within the E. coli isolates was evaluated using the Simpson's index of diversity. The diversity Simpson's index for the isolates was calculated to be 0.86 , showing a high level of genetic diversity among the E. coli isolates in this study. As shown in Table 4, clusters R4 and R6 - R11 included one E. coli isolate each one; however, only cluster R5 contained 8 isolates. Also, clusters R1 - R3 contained 4, 3 and 2 isolates, respectively. Ground meat isolates were just grouped in clusters R3 and R5. However, other isolates were also included in these clusters. B1 phylogroup strains were only grouped in the clusters R1 and R5. E. coli isolated from raw milk samples mostly were observed more resistant to antimicrobial agents than isolates from vegetable salad and ground meat samples. In this study, E. coli strains isolated from each food product were not grouped in a same RAPDPCR cluster and phylogroup, indicating a high level of genetic and phylogenetic diversity among the isolates from each food product. Also, as it can be seen in Table 4, mostly 
diverse patterns of antibiotic resistance can be found among the isolates from each food product.
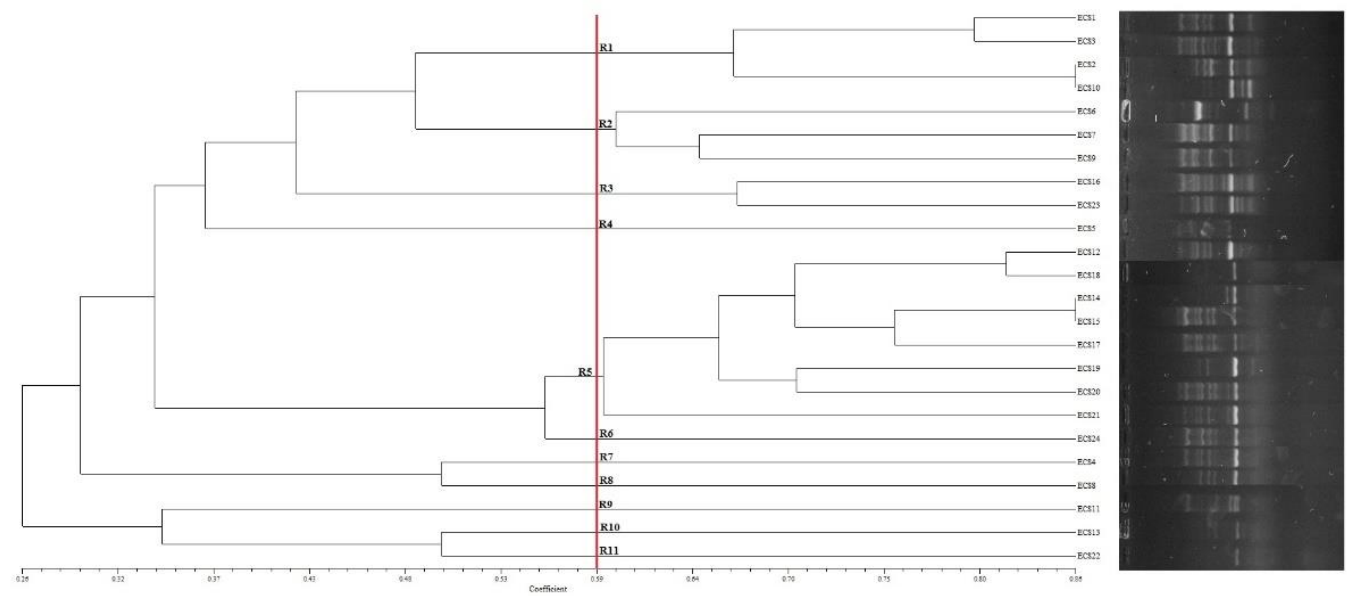

Figure 3. UPGMA Dendrogram with the Dice coefficient of E. coli isolates from food samples in the basis of RAPD-PCR analysis.

Table 4. Distribution of phylogroups among the E. coli isolates obtained from different food products.

\begin{tabular}{llllll}
\hline No. & Isolate & Food sample & Resistance phenotype ${ }^{a}$ & Phylogroup & RAPD-PCR type \\
\hline 1 & ECS1 & Vegetable salad & FOX, NIT & B1 & R1 \\
2 & ECS2 & Raw milk & AMC, IPM, AMX, TET, CHL, SXT & B1 & R1 \\
3 & ECS3 & Raw milk & AMC, KAN, AMX, TET, LVX, FEP, CHL, SXT & E & R1 \\
4 & ECS4 & Raw milk & SPT, AMC, AMX, AMP, TET, FEP, CHL, NIT, SXT & E & R7 \\
5 & ECS5 & Raw milk & FOX, AMC, KAN, AMX, TET, CHL, NIT, SXT & E & A \\
6 & ECS6 & Raw milk & AMC, CST, NIT, SXT & D & R2 \\
7 & ECS7 & Raw milk & KAN, TET, CST, CHL, SXT & A & R8 \\
8 & ECS8 & Vegetable salad & AMC, AMX, AMP, NIT & A & R2 \\
9 & ECS9 & Raw milk & SPT, AMC, KAN, AMX, AMP, TET, CHL, SXT & A & R1 \\
10 & ECS10 & Raw milk & FOX, SPT, AMX, AMP, GEN, NIT, SXT & R9 \\
11 & ECS11 & Raw milk & SPT, AMC, KAN, AMX, AMP, TET, CST, CHL, NIT SXT & D & R5 \\
12 & ECS12 & Raw milk & SPT, AMC, KAN, AMX, AMP, TET, CHL, NIT SXT & A & R10 \\
13 & ECS13 & Raw milk & FOX, AMX, NIT & A & R5 \\
14 & ECS14 & Ground meat & SPT, AMC, AMX, SXT & E & R5 \\
15 & ECS15 & Ground meat & SPT, AMC, AMX, CHL, SXT & D & R3 \\
16 & ECS16 & Ground meat & NAL, SPT, AMC, AMX, AMP, TET, CHL, SXT & B1 & R5 \\
17 & ECS17 & Vegetable salad & AMX. CHL, NIT, SXT & A & R5 \\
18 & ECS18 & Raw milk & AMX & A & R5 \\
19 & ECS19 & Vegetable salad & FEP & B1 & R5 \\
20 & ECS20 & Ground meat & AMX & B1 & E \\
21 & ECS21 & Raw milk & SPT, AMC, KAN, AMX, AMK, AMP, TET, CST, CHL, NIT, SXT & A & A \\
22 & ECS22 & Raw milk & SPT, AMC, KAN, AMX, AMP, TET, NIT, SXT & R11 \\
23 & ECS23 & Ground meat & SPT, AMX, TET, CHL, SXT & R3 \\
\hline
\end{tabular}

a NAL, nalidixic acid; FOX, cefoxitin; SPT, streptomycin; AMC, amoxicillin-clavulanic acid; IPM, imipenem; KAN, kanamycin; AMX, amoxicillin; AMK, amikacin; AMP, ampicillin; TET, tetracycline; LVX, levofloxacin; FEP, cefepime; GEN, gentamicin; CST, colistin; CHL, chloramphenicol; SXT, trimethoprim-sulfamethoxazole; NIT, nitrofurantoin.

\section{Discussion}

Most of E. coli strains attach and harmlessly colonize the human and animal colonal region of gastrointestinal tract and only seldomly cause mild to severe intestinal and extraintestinal diseases in immunocompromised individuals [7]. Conversely, diarrheal diseases caused by pathogenic E. coli are a severe public health problem and concern, a major cause of mortality and morbidity especially in children and infants [19]. Because of poor living conditions such as poor sanitation, environmental hygiene, and insuffi- 
cient education, diarrheal diseases with lethal outcomes mostly caused by different strains of $E$. coli are mainly prevailing in developing and low-income countries particularly in Asia, Africa, and Latin America [20]. Several studies reported that food animal origin might be an important source of human-acquired MDR pathogenic E. coli [17]. Poultry and meat products can be widely contaminated with pathogenic or nonpathogenic groups of E. coli of animal origins including MDR strains [21]. Because the main reservoir of this bacteria are intestinal tract and feces of warm-blooded animals and humans, the presence of E. coli strains (pathogenic or non-pathogenic) in foods, drinks, water and other environments has been used as an indicator of fecal contamination, poor hygiene and sanitation standards during production, processing, and distribution [22].

Few current studies are available regarding the prevalence rate E. coli strains in food products [18]. We aimed to provide determination of E. coli prevalence rate in food products including vegetable salad, raw milk, and ground meat samples collected from restaurants and local markets located in different areas Qazvin province, Iran. Total prevalence rate of E. coli was $6.95 \%$ (24 out of 345 samples) in this study which was significantly higher than that reported from Mexico (1.08\%; 56 out of 5162 food samples)[23], Colombia (2.1\%; 8 out of 380 food samples)[24], Korea $(2.2 \%$; 96 out of 4330 food samples)[25], Iran (4.0\%; 4 out of 100 samples)[26], and Japan (6.0\%; 20 out of 333 samples)[27]. Frequencies reported here were lower than those reported from China (11.0\%; 83 out of 750 total raw milk samples)[28] and Finland (43.0\%; 53 out of 120 food samples)[29]. The results of E. coli prevalence rate in this study indicated that poor hygiene and low standards of sanitation practices and behaviors have been employed during food production, processing, and distribution [27-29]. The results reported here also suggested that higher incidence rates of $E$. coli may not necessarily imply considerable $E$. coli contamination in food in Iran. Instead, using the efficient detection and identification methods might have led to significantly higher recovery of $E$. coli isolates from food samples $[22,26,30]$.

In this study, $54.16,20.83$, and $16.66 \%$ of all E. coli isolates (15, 5 and 4 out of 24 isolates) were isolated from raw cow milk, ground meat and vegetable salad samples, respectively. We found that the incidence rate of E. coli strains in raw milk samples was higher than that in vegetable salad and ground meat samples. Previously in Mexico, Canizalez-Roman et al. (2013) reported higher prevalence rate of diarrheagenic E. coli in dairy products $(28.4 \%)$, as the most contaminated food products, than that in meat $(24.0 \%)$, seafood and fish $(17.0 \%)$, prepared foods $(10.9 \%)$, and beverage and ice products (3.3\%) [23]. Recently, Ombarak et al. (2016) in Egypt demonstrated significantly higher incidence rate of E. coli contamination in raw milk samples $(76.4 \%)$ than that in Karish $(74.5 \%)$ and Ras $(21.7 \%)$ cheese samples among dairy products [30]. Raw milk could be considered as the most potential food vehicle of transmission for E. coli strains in Iran and around the world [31]. Higher prevalence rate and presence of E. coli strains in raw milk samples is an important indicator of poor hygiene practices, sanitation and fecal contamination in raw milk production and distribution. It is fairly reasonable to assume that the main source of E. coli contamination could be the consequence of human fecal contamination of those raw milk products during production or distribution activities [32].

One of the main concerns in food safety and public health is the emergence of antibiotic resistant foodborne bacterial pathogens. Resistance genes could be transferred from environments and animal food sources to the opportunistic pathogens, commensal microbiota and normal bacterial gut flora in human through food chains [33]. A wide range of antimicrobial agents are currently being employed worldwide for growth promotion, diseases prevention, and treatment of sick animals allowing the development of MDR foodborne pathogens [34]. A previous study in Korea reported high level of antibiotic resistance among E. coli strains isolated from food and food animals, finding that 15.6, 12.5, 10.4, 9.4, and 9.4\% of the E. coli isolates were resistant against tetracycline, streptomycin, ampicillin, ticarcillin and nalidixic acid antibiotics, respectively [25]. A 
study which was conducted in Mexico reported E. coli strains isolated from food samples resistant to tetracycline $(34 \% ; 19$ out of 56$)$, cefepime $(30 \% ; 17$ out of 56$)$ and ampicillin (29\%; 16 out of 56) [23]. A study in Iran by Mazaheri et al. (2014) reported high resistance to tetracycline and ampicillin in shiga-toxin producing E. coli (STEC) strains isolated from lettuce samples [26]. Another study which has been performed recently by Wang et al. (2017) showed that 49, 28, 24, 20 and 18\% of E. coli strains isolated from retail food samples were resistant to tetracycline, nalidixic acid, ampicillin, sulfamethoxazoletrimethoprim and cephalothin, respectively [27]. Moreover, Yu et al. (2020) in China isolated E. coli from raw milk samples with high resistance to penicillin, acetylspiramycin, oxacillin, lincomycin, sulphamethoxazole, cephalosporin and ampicillin [28]. Recent study of Elmonir et al. (2021) in Egypt also showed a high resistance to nalidixic acid, ampicillin and streptomycin in STEC strains isolated from raw milk and beef samples [35].

E. coli isolates in this study were resistant to amoxicillin $(79.16 \%)$, trimethoprimsulfamethoxazole $(70.83 \%)$, amoxicillin-clavulanic acid $(62.5 \%)$, tetracycline $(54.16 \%)$, chloramphenicol (54.16\%), nitrofurantoin (54.16\%), ampicillin (45.83\%), streptomycin $(45.83 \%)$ and kanamycin (33.33\%). All isolates were completely susceptible to norfloxacin and azithromycin. We also found that E. coli isolates from raw milk samples were more resistant to antibiotics than isolates from vegetable salad and ground meat samples. Obviously, resistance to different classes of antimicrobial agents among the E. coli isolates from food products have recently increased worldwide [18,28,35]. From the regional point of view, antibiotic resistance patterns of E. coli isolates from different food products at the present study are partly in agreement with previous studies in Iran [26]. In this study, $70.83 \%$ (17 out of 24 ) of $E$. coli isolates expressed resistance to at least three different classes of antibiotics and were regarded as MDR E. coli strains. The MDR rates of E. coli isolated from food samples reported in this study was significantly higher than that reported in Korea (12.5\%) [25], Turkey (20\%) [36], and Egypt (51.42\%) [35], and lower than that reported in China (100\%) [28] and Mexico (92.4\%) [23]. The continuous global resistance among $E$. coli strains of food origins have been considered a serious threat to the public health and a major concern in food safety [16]. Indiscriminate and irrational use of antibiotics in the water and feeds of lifestock (for infection treatment and/or growth promotion) may contribute to multidrug resistance in E. coli strains. $[18,27,28]$. This may be regarded as the main reason for high prevalence of MDR E. coli isolates from food samples in this study.

Approximately, only 2000 of the 4721 genes in E. coli are conserved. The high level of genomic plasticity, including gaining and losing genes, in the rest of these genes contributed to developing a simple and inexpensive assay of genomic classification of E. coli strains to assign these isolates to specific phylogenetic groups [11]. E. coli strains of the same phylogenetic group share similar phenotypic and genotypic characterizations, disease causing ability, life history features and ecological attributes [37]. Different phylogenetic groups of $E$. coli have been found in specific hosts and demonstrated the same level of adaptability to the environmental conditions [11]. Of the $24 \mathrm{E}$. coli isolates from food samples in this study, phylogenetic group A was the most prevalent $(46 \%$; 11 out of 24) and phylogroup D was the least common. The results of our study differ from another study has previously been conducted by Higgins et al. (2007) in the USA and they show that 26,25 and $17 \%$ of the E. coli isolates from animal, humans, and water were belonging to the phylogroups B1, A and D, respectively [38]. Most E. coli isolates belonging to the phylogroups B2 and lesser to D carry more virulence encoding gene factors than other phylogroups members and contribute to extra-intestinal diseases; however, some strains included in other phylogroups (A and B1) have been identified as causes of diarrheal diseases in humans [37]. Also, several studies showed the phylogroup B1 strains were more resistant to inappropriate environmental conditions [39]. In our study, we have not isolated any E. coli strain belonging to the phylogroup B2 indicating low extra intestinal pathogenic potentiality of the E. coli isolates from food products. 
Many DNA fingerprinting assays such as ERIC, BOX and RAPD-PCR methods have widely been used to evaluate the clonal relatedness and genetic diversity among foodborne pathogens isolated from different food sources [40]. In this study, we used RAPD-PCR to assess the genetic diversity and clonality of E. coli strains isolated from vegetable salad, raw milk and ground meat samples as previous studies showed for clinical and foodborne pathogen isolates [41]. The results of RAPD-PCR with the primer UBC245 on E. coli isolates demonstrated a major cluster (cluster R5) with 8 members including $33.3 \%$ of total E. coli isolates. In this study, a high level of genetic diversity was found among the isolates. Isolates from ground meat samples were grouped in the same clusters (R3 and R5) and the E. coli isolates belonging to the phylogenetic group B1 were also grouped in the same clusters (R1 and R5). Our RAPD-PCR analysis suggested that antibiotic resistance patterns are randomly distributed among all 11 genetic clusters. Vidovic and Korber (2006) used RAPD-PCR assay to evaluate genetic diversity among the E. coli serotype O157: H7 isolates from feedlot cattle. They found a high level of genetic diversity and wide distribution of antibiotic resistance patterns among the isolates [42]. The greater level of diversity of genotypic and phenotypic properties among the E. coli isolates in this and previous studies could be due to some important factors including inter-species transfer of E. coli strains from domestic or farm animals to human and vice versa, consuming foods and drinks contaminated with foodborne pathogens, long duration of sample collection period and international travels [43,44].

\section{Materials and Methods}

4.1. Collection of food samples

A total number of 345 food samples including vegetable salad $(n=115)$, raw cow milk ( $n=115)$, and ground meat samples $(n=115)$ were purchased and collected from 27 restaurants and 45 local markets located in different areas throughout the Qazvin province, Iran, between August 2018 and February 2019. All samples were aseptically collected in sterile tubes and containers, immediately transported to the food microbiology laboratory in cool boxes with ice packs for further analysis.

4.2. Isolation and identification of E. coli

E. coli was isolated and identified in food samples according to the method previously described by Ombarak et al. (2016) [30]. $10 \mathrm{~mL}$ of raw milk, $25 \mathrm{gr}$ of vegetable salad and 25 gr of ground meat samples were mixed with either 90, or $225 \mathrm{~mL}$ of tryptic soy broth (TSB, Promedia, Spain) yielding a 1:10 sample dilution, then homogenized at $400 \mathrm{rpm}$ for $10 \mathrm{~min}$ using a Stomacher-blender BagMixer (InterScience, France) and incubated at $37^{\circ} \mathrm{C}$ for $16 \mathrm{~h} .100 \mu \mathrm{L}$ of the enrichment cultures were streaked onto eosin methylene blue agar (EMB, Promedia, Spain) plates and incubated at $37^{\circ} \mathrm{C}$ for $24 \mathrm{~h}$. Presumptive E. coli colonies on each EMB agar plate (blue-black colonies with a metallic green sheen) were selected, picked and subjected to Gram-staining and biochemical tests including motility, oxidase, indole production, citrate utilization, methyl red, VogesProskauer, triple sugar iron, urease, lysine and recommended sugar fermentation tests (Promedia, Spain). All confirmed E. coli isolates were stocked in TSB (Promedia, Spain) medium containing $18 \%(\mathrm{v} / \mathrm{v})$ glycerol, incubated at $37^{\circ} \mathrm{C}$ for $24 \mathrm{~h}$ and kept at $-80{ }^{\circ} \mathrm{C}$ until further analysis. Escherichia coli ATCC 25922 (Serotype O6) a recommended reference strain for antibiotic susceptibility testing was used as positive control [45]. The control strain was activated by inoculation into TSB medium and incubation at $37^{\circ} \mathrm{C}$ for $24 \mathrm{~h}$.

4.3. Antimicrobial resistance testing

Antimicrobial susceptibility testing for E. coli isolates was performed using a KirbyBauer disk diffusion assay based on the standards and interpretive criteria previously established and developed by Clinical and Laboratory Standards Institute [46]. Nineteen commercial antibiotic disks (Oxoid, UK) used in this study included cefepime (FEP), 30 $\mu \mathrm{g}$; cefoxitin (FOX), $30 \mu \mathrm{g}$; kanamycin (KAN), $30 \mu \mathrm{g}$; ampicillin (AMP), $10 \mu \mathrm{g}$; imipenem (IPM), $10 \mu \mathrm{g}$; amoxicillin (AMX), $25 \mu \mathrm{g}$; amoxicillin-clavulanic acid (AMC), 20/10 $\mu \mathrm{g}$; streptomycin (SPT), $10 \mu \mathrm{g}$; amikacin (AMK), $30 \mu \mathrm{g}$; norfloxacin (NOR), $10 \mu \mathrm{g}$; gentamicin (GEN), $10 \mu \mathrm{g}$; nalidixic acid (NAL), $30 \mu \mathrm{g}$; levofloxacin (LVX), $5 \mu \mathrm{g}$; colistin (CST) 10 
$\mu \mathrm{g}$; azithromycin (AZM), $15 \mu \mathrm{g}$; tetracycline (TET), $30 \mu \mathrm{g}$; chloramphenicol (CHL), 30 $\mu \mathrm{g}$; trimethoprim-sulfamethoxazole (SXT), 1.25/23.75 $\mu \mathrm{g}$, and nitrofurantoin (NIT), 300 $\mu \mathrm{g}$. The results of antibiotic resistance phenotypes were recorded and interpreted according to CLSI guidelines [46]. Klebsiella pneumoniae ATCC 700603, Escherichia coli ATCC 25922 and Staphylococcus aureus ATCC 25923 were used as the reference strains for quality control.

\subsection{DNA extraction}

All isolates and the control strain (E. coli ATCC 25922) were grown on bovine heart infusion (BHI, Promedia, Spain) broth overnight at $37^{\circ} \mathrm{C} .1 \mathrm{~mL}$ of Phosphate Buffered Saline (PBS, Promedia Spain) was mixed with $1 \mathrm{~mL}$ of the bacterial suspension and centrifuged at $8000 \mathrm{~g}$ for $4 \mathrm{~min}$. The supernatant was removed, and the bacterial sediment was subjected to genome extraction using Sinaclon bacterial DNA extraction kit (Sinaclon Co., Iran) according to the manufacturer's instruction. Quantity and quality of the extracted DNA were measured using NanoDrop 2000 spectrophotometer (Thermo Scientific, USA). The concentrations of the extracted DNA were adjusted to $50 \mu \mathrm{g} / \mathrm{mL}$ with PBS prior to PCR reactions.

\subsection{Phylogroups determination}

To determine phylogroups in the E. coli isolates, a triplex PCR method described by Clermont et al. (2000) was used [10]. PCR was carried out in an ABI PCR thermal cycler model 9092 (Applied Biosystems, USA). Specific primers which have previously been described by Clermont et al. (2000) were used to amplify TSPE4, yjaA and chuA genes [10]. PCRs were performed in $20-\mu \mathrm{L}$ reaction volumes containing $10 \mu \mathrm{L}$ of PCR Master Mix kit (Ampliqon, Denmark), $0.5 \mu \mathrm{L}$ of each primer $(2 \mu \mathrm{M} / \mu \mathrm{L}), 2 \mu \mathrm{L}$ of DNA template and nuclease-free deionized water to reach the final reaction volume. The PCR reaction was performed as follows: initial denaturation step at $95{ }^{\circ} \mathrm{C}$ for $5 \mathrm{~min}$, following by 35 cycles comprised $95{ }^{\circ} \mathrm{C}$ for $30 \mathrm{~s}, 59^{\circ} \mathrm{C}$ for $30 \mathrm{~s}, 72{ }^{\circ} \mathrm{C}$ for $40 \mathrm{~s}$; and a final extension at 72 ${ }^{\circ} \mathrm{C}$ for $5 \mathrm{~min}$. Amplified PCR products were separated and characterized using gel electrophoresis on a 1.5\% (w/v) agarose gel containing DNA safe stain (Invitrogen, UK) at $110 \mathrm{v}$ for $1 \mathrm{~h}$. Gels were visualized and the phylogroup patterns were recorded using Novin-Pars Gel Documentation system (NovinPars Co., Iran). Non-pathogenic E. coli ATCC 25922 containing all three genes was used as the control strain.

\subsection{RAPD-PCR genotyping}

In this study, the genetic diversity among the E. coli isolates from food products was evaluated using RAPD-PCR assay as previously described. The primer UBC245: 5'CGCGTGCCAG -3', was used for RAPD-PCR [47]. The PCR was performed in $25-\mu \mathrm{L}$ reaction volumes containing $12.5 \mu \mathrm{L}$ of PCR Master Mix (Ampliqon, Denmark), $1 \mu \mathrm{L}$ of UBC245 primer $(5 \mathrm{pM} / \mu \mathrm{L}), 2 \mu \mathrm{L}$ of DNA template and sterilized deionized water to reach the final reaction volume. The PCR was carried out as follows: 6 min at $95{ }^{\circ} \mathrm{C}, 1$ min at $36{ }^{\circ} \mathrm{C}, 4 \mathrm{~min}$ at $72{ }^{\circ} \mathrm{C}$ and 35 cycles of $95^{\circ} \mathrm{C}$ for $1 \mathrm{~min}, 36^{\circ} \mathrm{C}$ for $1 \mathrm{~min}$, and $72{ }^{\circ} \mathrm{C}$ for $4 \mathrm{~min}$. The amplicons were separated and characterized using gel electrophoresis on a $1 \%(\mathrm{w} / \mathrm{v})$ agarose gel containing DNA safe stain (Invitrogen, UK) at $90 \mathrm{v}$ for $2 \mathrm{~h}$. Gels were visualized, and the RAPD patterns were recorded by Novin-Pars Gel Documentation system (NovinPars Co., Iran). PyElph software version 1.4 [48] was used for analysis of RAPD genetic markers. The RAPD-PCR dendrogram was constructed based on UPGMA method and Dice coefficient using NTSYS-pc version 2.1 [49] software. The RAPD-PCR patterns of the E. coli isolates with a similarity index higher than 0.59 were regarded as the closely related RAPD-PCR pattern type.

4.7. Statistical analysis

Fisher's exact and Chi-square tests were used to evaluate significant differences ( $\mathrm{P}<$ 0.05) between the incidence rates using SPSS version 21.0.1 (IBM Corp., NY, USA) software. All experimental and measurements were performed in triplicates.

\section{Conclusions}

In conclusion, the study investigates the prevalence rate, antimicrobial susceptibility, phylogenetic groups and genetic diversity of E. coli strains isolated from food prod- 
ucts including raw milk, vegetable salad, and ground meat samples. Our results demonstrated that prevalence the rate of $E$. coli was higher in raw milk samples than in vegetable salad and ground meat samples. This indicates poor hygiene and sanitation practices and fecal contamination during raw milk production and distribution. This study showed that E. coli isolates were highly resistant to amoxicillin, trimethoprimsulfamethoxazole, amoxicillin-clavulanic acid, tetracycline, chloramphenicol, nitrofurantoin, ampicillin, streptomycin, and kanamycin as well as completely sensitive to norfloxacin and azithromycin antibiotics. We found that $70.83 \%$ of E. coli isolates were MDR to at least three classes of antimicrobial agents. Irrational and indiscriminate use of clinical and veterinary antibiotics in livestock feeds and water fed may contribute to multidrug resistance in E. coli strains of food origin which is becoming a serious concern of public health. Most E. coli isolates (46\%) belonged to phylogroup A. High level of genetic diversity was found among the isolates. RAPD-PCR with UBC245 primer discriminated the E. coli isolates into 11 genetic clusters. $33.3 \%$ of all isolates were grouped in a major cluster (R5). Isolates from ground meat samples were grouped in the same clusters. Also, the E. coli isolates belonging to the phylogenetic group B1 were grouped in the same clusters. Antimicrobial resistance patterns are randomly distributed among the clusters. A cognitive scientific approach is strongly necessary to improve public health and prevent foodborne diseases. Novel and efficient food safety control and surveillance systems of foodborne pathogens especially MDR strains in developing and low-income countries is strongly required to control and prevent foodborne pathogen contamination and diseases.

Author Contributions: Conceptualization, B.P. and W.M.B.; methodology, B.P., S.A. and Z.A.; software, B.P. and W.M.B.; validation, B.P. and R.M.; formal analysis, B.P., S.A., Z.A. and A.P.; investigation, B.P.; resources, R.M. and A.P.; data curation, B.P.; writing-original draft preparation, B.P.; writing - review and editing, W.M.B; visualization, B.P.; supervision, R.M. and A.P.; project administration, B.P.; All authors have read and agreed to the published version of the manuscript.

Funding: This research received no external funding.

Institutional Review Board Statement: Not applicable.

Informed Consent Statement: Not applicable.

Data Availability Statement: We confirm that all data supporting the findings of this study are available within the article.

Acknowledgments: We thank and appreciate our colleagues and technicians in Department of Food Safety and Hygiene, Qazvin University of Medical Science who assisted us in this project.

Conflicts of Interest: The authors declare no conflict of interest.

\section{References}

1. Smith, J.L.; Fratamico, P.M. Emerging and re-emerging foodborne pathogens. Foodborne Pathogens and Disease 2018, 15, 737757.

2. Dewey-Mattia, D.; Manikonda, K.; Hall, A.J.; Wise, M.E.; Crowe, S.J. Surveillance for foodborne disease outbreaks -United States, 2009-2015. MMWR Surveillance Summaries 2018, 67, 1.

3. Organization, W.H. WHO estimates of the global burden of foodborne diseases: foodborne disease burden epidemiology reference group 2007-2015; World Health Organization: 2015.

4. Devleesschauwer, B.; Haagsma, J.A.; Mangen, M.-J.J.; Lake, R.J.; Havelaar, A.H. The global burden of foodborne disease. In Food safety economics; Springer: 2018; pp. 107-122.

5. Mainil, J. Escherichia coli virulence factors. Veterinary immunology and immunopathology 2013, 152, 2-12.

6. Tenaillon, O.; Skurnik, D.; Picard, B.; Denamur, E. The population genetics of commensal Escherichia coli. Nature reviews microbiology 2010, 8, 207-217.

7. Croxen, M.A.; Finlay, B.B. Molecular mechanisms of Escherichia coli pathogenicity. Nature Reviews Microbiology 2010, 8, 2638.

8. Ercumen, A.; Pickering, A.J.; Kwong, L.H.; Arnold, B.F.; Parvez, S.M.; Alam, M.; Sen, D.; Islam, S.; Kullmann, C.; Chase, C. Animal feces contribute to domestic fecal contamination: evidence from E. coli measured in water, hands, food, flies, and soil in Bangladesh. Environmental science \& technology 2017, 51, 8725-8734.

9. Jang, J.; Hur, H.G.; Sadowsky, M.J.; Byappanahalli, M.; Yan, T.; Ishii, S. Environmental Escherichia coli: ecology and public health implications - a review. Journal of applied microbiology 2017, 123, 570-581. 
10. Clermont, O.; Bonacorsi, S.; Bingen, E. Rapid and simple determination of the Escherichia coli phylogenetic group. Applied and environmental microbiology 2000, 66, 4555-4558.

11. Clermont, O.; Christenson, J.K.; Denamur, E.; Gordon, D.M. The C lermont E scherichia coli phylo-typing method revisited: improvement of specificity and detection of new phylo-groups. Environmental microbiology reports 2013, 5, 58-65.

12. Beghain, J.; Bridier-Nahmias, A.; Le Nagard, H.; Denamur, E.; Clermont, O. ClermonTyping: an easy-to-use and accurate in silico method for Escherichia genus strain phylotyping. Microbial genomics 2018, 4.

13. Stoppe, N.d.C.; Silva, J.S.; Carlos, C.; Sato, M.I.; Saraiva, A.M.; Ottoboni, L.M.; Torres, T.T. Worldwide phylogenetic group patterns of Escherichia coli from commensal human and wastewater treatment plant isolates. Frontiers in microbiology 2017, 8, 2512.

14. Caniça, M.; Manageiro, V.; Abriouel, H.; Moran-Gilad, J.; Franz, C.M. Antibiotic resistance in foodborne bacteria. Trends in Food Science \& Technology 2019, 84, 41-44.

15. Walsh, C.; Fanning, S. Antimicrobial resistance in foodborne pathogens-A cause for concern? Current drug targets 2008, 9 , 808-815.

16. Doyle, M.E. Multidrug-resistant pathogens in the food supply. Foodborne Pathogens and Disease 2015, 12, 261-279.

17. Petty, N.K.; Zakour, N.L.B.; Stanton-Cook, M.; Skippington, E.; Totsika, M.; Forde, B.M.; Phan, M.-D.; Moriel, D.G.; Peters, K.M.; Davies, M. Global dissemination of a multidrug resistant Escherichia coli clone. Proceedings of the National Academy of Sciences 2014, 111, 5694-5699.

18. Ramadan, H.; Jackson, C.R.; Frye, J.G.; Hiott, L.M.; Samir, M.; Awad, A.; Woodley, T.A. Antimicrobial resistance, genetic diversity and multilocus sequence typing of Escherichia coli from humans, retail chicken and ground beef in Egypt. Pathogens 2020, 9, 357.

19. Gomes, T.A.; Elias, W.P.; Scaletsky, I.C.; Guth, B.E.; Rodrigues, J.F.; Piazza, R.M.; Ferreira, L.C.; Martinez, M.B. Diarrheagenic escherichia coli. Brazilian Journal of Microbiology 2016, 47, 3-30.

20. Beauchamp, C.S.; Sofos, J.N. Diarrheagenic Escherichia coli. Pathogens and toxins in foods: challenges and interventions 2009, 71-94.

21. Alhashash, F.; Weston, V.; Diggle, M.; McNally, A. Multidrug-resistant Escherichia coli bacteremia. Emerging infectious diseases 2013, 19, 1699.

22. 22. Pormohammad, A.; Nasiri, M.J.; Azimi, T. Prevalence of antibiotic resistance in Escherichia coli strains simultaneously isolated from humans, animals, food, and the environment: a systematic review and meta-analysis. Infection and drug resistance 2019, 12, 1181.

23. Canizalez-Roman, A.; Gonzalez-Nuñez, E.; Vidal, J.E.; Flores-Villaseñor, H.; León-Sicairos, N. Prevalence and antibiotic resistance profiles of diarrheagenic Escherichia coli strains isolated from food items in northwestern Mexico. International journal of food microbiology 2013, 164, 36-45.

24. Rúgeles, L.C.; Bai, J.; Martínez, A.J.; Vanegas, M.C.; Gómez-Duarte, O.G. Molecular characterization of diarrheagenic Escherichia coli strains from stools samples and food products in Colombia. International journal of food microbiology 2010, 138, 282-286.

25. Lee, G.Y.; Jang, H.I.; Hwang, I.G.; Rhee, M.S. Prevalence and classification of pathogenic Escherichia coli isolated from fresh beef, poultry, and pork in Korea. International journal of food microbiology 2009, 134, 196-200.

26. Mazaheri, S.; Ahrabi, S.S.; Aslani, M.M. Shiga toxin-producing Escherichia coli isolated from lettuce samples in Tehran, Iran. Jundishapur journal of microbiology 2014, 7.

27. Wang, L.; Nakamura, H.; Kage-Nakadai, E.; Hara-Kudo, Y.; Nishikawa, Y. Prevalence, antimicrobial resistance and multiplelocus variable-number tandem-repeat analysis profiles of diarrheagenic Escherichia coli isolated from different retail foods. International journal of food microbiology 2017, 249, 44-52.

28. Yu, Z.; Wang, J.; Ho, H.; Wang, Y.; Huang, S.; Han, R. Prevalence and antimicrobial-resistance phenotypes and genotypes of Escherichia coli isolated from raw milk samples from mastitis cases in four regions of China. Journal of global antimicrobial resistance 2020, 22, 94-101.

29. 29. Kagambega, A.; Martikainen, O.; Siitonen, A.; Traore, A.S.; Barro, N.; Haukka, K. Prevalence of diarrheagenic E scherichia coli virulence genes in the feces of slaughtered cattle, chickens, and pigs in B urkina F aso. MicrobiologyOpen 2012, 1, 276-284.

30. Ombarak, R.A.; Hinenoya, A.; Awasthi, S.P.; Iguchi, A.; Shima, A.; Elbagory, A.-R.M.; Yamasaki, S. Prevalence and pathogenic potential of Escherichia coli isolates from raw milk and raw milk cheese in Egypt. International Journal of Food Microbiology 2016, 221, 69-76

31. Berge, A.; Baars, T. Raw milk producers with high levels of hygiene and safety. Epidemiology \& Infection 2020, 148.

32. Boor, K.J.; Wiedmann, M.; Murphy, S.; Alcaine, S. A 100-year review: microbiology and safety of milk handling. Journal of Dairy Science 2017, 100, 9933-9951.

33. Oniciuc, E.-A.; Likotrafiti, E.; Alvarez-Molina, A.; Prieto, M.; López, M.; Alvarez-Ordóñez, A. Food processing as a risk factor for antimicrobial resistance spread along the food chain. Current Opinion in Food Science 2019, 30, 21-26.

34. Hudson, J.A.; Frewer, L.J.; Jones, G.; Brereton, P.A.; Whittingham, M.J.; Stewart, G. The agri-food chain and antimicrobial resistance: A review. Trends in Food Science \& Technology 2017, 69, 131-147.

35. Elmonir, W.; Shalaan, S.; Tahoun, A.; Mahmoud, S.F.; Remela, E.M.A.; Eissa, R.; El-Sharkawy, H.; Shukry, M.; Zahran, R.N. Prevalence, antimicrobial resistance, and genotyping of Shiga toxin-producing Escherichia coli in foods of cattle origin, diarrheic cattle, and diarrheic humans in Egypt. Gut Pathogens 2021, 13, 1-11. 
36. Onmaz, N.E.; Yildirim, Y.; Karadal, F.; Hizlisoy, H.; Al, S.; Gungor, C.; Disli, H.B.; Barel, M.; Dishan, A.; Tegin, R.A.A. Escherichia coli $\mathrm{O} 157$ in fish: Prevalence, antimicrobial resistance, biofilm formation capacity, and molecular characterization. LWT 2020, 133, 109940.

37. Bailey, J.K.; Pinyon, J.L.; Anantham, S.; Hall, R.M. Distribution of human commensal Escherichia coli phylogenetic groups. Journal of clinical microbiology 2010, 48, 3455-3456.

38. Higgins, J.; Hohn, C.; Hornor, S.; Frana, M.; Denver, M.; Joerger, R. Genotyping of Escherichia coli from environmental and animal samples. Journal of microbiological methods 2007, 70, 227-235.

39. Corzo-Ariyama, H.A.; García-Heredia, A.; Heredia, N.; García, S.; León, J.; Jaykus, L.; Solís-Soto, L. Phylogroups, pathotypes, biofilm formation and antimicrobial resistance of Escherichia coli isolates in farms and packing facilities of tomato, jalapeño pepper and cantaloupe from Northern Mexico. International journal of food microbiology 2019, 290, 96-104.

40. Yoke-Kqueen, C.; Teck-Ee, K.; Son, R.; Yoshitsugu, N.; Mitsuaki, N. Molecular characterisation of Vibrio parahaemolyticus carrying tdh and trh genes using ERIC-, RAPD-and BOX-PCR on local Malaysia bloody clam and Lala. International Food Research Journal 2013, 20, 3299.

41. Salehi, T.Z.; Madani, S.A.; Karimi, V.; Khazaeli, F.A. Molecular genetic differentiation of avian Escherichia coli by RAPD-PCR. Brazilian Journal of Microbiology 2008, 39, 494-497.

42. Vidovic, S.; Korber, D.R. Prevalence of Escherichia coli O157 in Saskatchewan cattle: characterization of isolates by using random amplified polymorphic DNA PCR, antibiotic resistance profiles, and pathogenicity determinants. Applied and environmental microbiology 2006, 72, 4347-4355.

43. Rousset, F.; Cabezas-Caballero, J.; Piastra-Facon, F.; Fernández-Rodríguez, J.; Clermont, O.; Denamur, E.; Rocha, E.P.; Bikard, D. The impact of genetic diversity on gene essentiality within the Escherichia coli species. Nature Microbiology 2021, 6, 301312.

44. Sahilah, A.; Audrey, L.; Ong, S.; Wan Sakeenah, W.; Saiyyah, S.; Norrakiah, A.; Aminah, A.; Ahmad Azuhairi, A. DNA profiling among egg and beef meat isolates of Escherichia coli by enterobacterial repetitive intergenic consensus-PCR (ERIC-PCR) and random amplified polymorphic DNA-PCR (RAPD-PCR). International Food Research Journal 2010, 17.

45. Minogue, T.; Daligault, H.; Davenport, K.; Bishop-Lilly, K.; Broomall, S.; Bruce, D.; Chain, P.; Chertkov, O.; Coyne, S.; Freitas, T. Complete genome assembly of Escherichia coli ATCC 25922, a serotype O6 reference strain. Genome announcements 2014, 2 .

46. Humphries, R.M.; Ambler, J.; Mitchell, S.L.; Castanheira, M.; Dingle, T.; Hindler, J.A.; Koeth, L.; Sei, K. CLSI methods development and standardization working group best practices for evaluation of antimicrobial susceptibility tests. Journal of clinical microbiology 2018, 56.

47. 47. Berthold-Pluta, A.; Garbowska, M.; Stefańska, I.; Pluta, A. Microbiological quality of selected ready-to-eat leaf vegetables, sprouts and non-pasteurized fresh fruit-vegetable juices including the presence of Cronobacter spp. Food microbiology 2017, 65, 221-230.

48. Pavel, A.B.; Vasile, C.I. PyElph-a software tool for gel images analysis and phylogenetics. BMC bioinformatics 2012, 13, 1-6.

49. Rohlf, F. NTSYS-pc numeral taxonomy and multivariate analysis system, version $2.10 \mathrm{j}$. New York, NY: Exeter Publications 2001. 